International Journal of Pure and Applied Mathematics

Volume 89 No. 2 2013, 141-146

ISSN: 1311-8080 (printed version); ISSN: 1314-3395 (on-line version)

url: http://www.ijpam.eu

doi: http://dx.doi.org/10.12732/ijpam.v89i2.2

ijpam.eu

\title{
A NEW BOUND ON POINTWISE POISSON APPROXIMATION FOR RANDOM SUMS OF BERNOULLI RANDOM VARIABLES
}

\author{
K. Teerapabolarn \\ Department of Mathematics \\ Faculty of Science \\ Burapha University \\ Chonburi, 20131, THAILAND
}

\begin{abstract}
A new bound for the point metric between the distribution of random sums of independent Bernoulli random variables and an appropriate Poisson distribution is obtained. The bound in this study is sharper than those reported in [3].
\end{abstract}

AMS Subject Classification: 62E17, 60F05, 60G05

Key Words: Bernoulli random variable, point metric, Poisson approximation, random sums, Stein-Chen method

\section{Introduction}

Let $X_{1}, X_{2}, \ldots$ be a sequence of independent Bernoulli random variables, each with probability $p_{i}=P\left(X_{i}=1\right)=1-P\left(X_{i}=0\right)$, and let $N$ be a nonnegative integer-valued random variable and independent of the $X_{i}$ 's. Let us consider the sum $S_{N}=\sum_{i=1}^{N} X_{i}$, which is called random sums. In the case of $N=n(n \in \mathbb{N})$, is fixed, there has been some research related to the pointwise approximation of the distribution of the sum $S_{n}$ by Poisson distribution, which can be found in [4], [6] and [5]. Let $\lambda_{N}=\sum_{i=1}^{N} p_{i}, \lambda=E\left(\lambda_{N}\right)$ and $U_{\lambda}$ a Poisson

Received: May 22, 2013

(c) 2013 Academic Publications, Ltd. url: www.acadpubl.eu 
random variable with mean $\lambda$. For approximating the distribution of $S_{N}$ by a Poisson distribution with mean $\lambda$ in the point metric form, Yannaros [8] gave a bound in the form of

$$
\left|P\left(S_{N}=x_{0}\right)-P\left(U_{\lambda}=x_{0}\right)\right| \leq E\left|\lambda_{N}-\lambda\right|+E\left(\frac{1-e^{-\lambda_{N}}}{\lambda_{N}} \sum_{i=1}^{N} p_{i}^{2}\right),
$$

where $x_{0} \in \mathbb{N}$. In this case, Kongudomthrap and Chaidee [3] gave a bound in the form of

$$
\left|P\left(S_{N}=x_{0}\right)-P\left(U_{\lambda}=x_{0}\right)\right| \leq \frac{7 \lambda}{2 x_{0}}
$$

and

$$
\left|P\left(S_{N}=x_{0}\right)-P\left(U_{\lambda}=x_{0}\right)\right| \leq \frac{3 \lambda}{2}+2 \min \left\{\lambda, E\left|\lambda_{N}-\lambda\right|\right\}
$$

where the probability $P\left(S_{N}=0\right)=E\left[\prod_{i=1}^{N}\left(1-p_{i}\right)\right]$.

In this study, we focus on improving the bounds in (1.1)-(1.3) by using the stein-Chen method, and we also describe this method in Section 2. In Section 3, we use the Stein-Chen method to determine a new bound for this point metric. Concluding remarks are presented in the last section.

\section{Method}

The Stein-Chen was first developed and applied in the Poisson approximation by Chen [2]. Stein's equation for Poisson distribution with mean $\lambda>0$ is, for given $h$, of the form

$$
h(x)-\mathcal{P}_{\lambda}(h)=\lambda f(x+1)-x f(x)
$$

where $\mathcal{P}_{\lambda}(h)=e^{-\lambda} \sum_{l=0}^{\infty} h(l) \frac{\lambda^{l}}{l !}$ and $f$ and $h$ are bounded real valued functions defined on $\mathbb{N} \cup\{0\}$. For $\Omega \subseteq \mathbb{N} \cup\{0\}$, let function $h_{\Omega}: \mathbb{N} \cup\{0\} \rightarrow \mathbb{R}$ be defined by

$$
h_{\Omega}(x)= \begin{cases}1 & \text { if } x \in \Omega \\ 0 & \text { if } x \notin \Omega\end{cases}
$$


Let $C_{x}=\{0, \ldots, x\}$. For $\Omega=\left\{x_{0}\right\}$ where $x_{0} \in \mathbb{N} \cup\{0\}$, following [1] and writing $h_{x_{0}}=h_{\left\{x_{0}\right\}}$, the solution $f_{x_{0}}=f_{\left\{x_{0}\right\}}$ of $(2.1)$ is of the form

$$
f_{x_{0}}(x)= \begin{cases}-(x-1) ! \lambda^{-x} e^{\lambda}\left[\mathcal{P}_{\lambda}\left(h_{x_{0}}\right) \mathcal{P}_{\lambda}\left(h_{C_{x-1}}\right)\right] & \text { if } x \leq x_{0} \\ (x-1) ! \lambda^{-x} e^{\lambda}\left[\mathcal{P}_{\lambda}\left(h_{x_{0}}\right) \mathcal{P}_{\lambda}\left(1-h_{C_{x-1}}\right)\right] & \text { if } x>x_{0} \\ 0 & \text { if } x=0\end{cases}
$$

Let $\Delta f_{x_{0}}(x)=f_{x_{0}}(x+1)-f_{x_{0}}(x)$, then the following lemma gives bounds for $\left|f_{x_{0}}\right|$ and $\left|\Delta f_{x_{0}}\right|$.

Lemma 2.1. Let $x_{0}, x \in \mathbb{N}$, then we have the following:

$$
\sup _{x \geq 1}\left|f_{x_{0}}(x)\right| \leq \min \left\{\frac{1-e^{-\lambda}}{\lambda}, \frac{1}{x_{0}}\right\}[7]
$$

and

$$
\sup _{x \geq 1}\left|\Delta f_{x_{0}}(x)\right| \leq \min \left\{\frac{1-e^{-\lambda}}{\lambda}, \frac{1}{x_{0}}\right\}[1] .
$$

\section{Results}

The following theorem presents a new bound for the point metric between the distributions of $S_{N}$ and $U_{\lambda}$, which is the desired result.

Theorem 3.1. For $x_{0} \in \mathbb{N}$, then we have

$$
\begin{aligned}
\left|P\left(S_{N}=x_{0}\right)-P\left(U_{\lambda}=x_{0}\right)\right| \leq \min \left\{\frac{1-e^{-\lambda}}{\lambda}, \frac{1}{x_{0}}\right\} E\left|\lambda_{N}-\lambda\right| \\
+\min \left\{E\left(\frac{1-e^{-\lambda_{N}}}{\lambda_{N}} \sum_{i=1}^{N} p_{i}^{2}\right), \frac{1}{x_{0}} E\left(\sum_{i=1}^{N} p_{i}^{2}\right)\right\} .
\end{aligned}
$$

Proof. It follows the fact that

$$
\begin{aligned}
\left|P\left(S_{N}=x_{0}\right)-P\left(U_{\lambda}=x_{0}\right)\right| & \leq \sum_{n=0}^{\infty} P(N=n)\left|P\left(S_{n}=x_{0}\right)-P\left(U_{\lambda}=x_{0}\right)\right| \\
& \leq \sum_{n=0}^{\infty} P(N=n)\left|P\left(S_{n}=x_{0}\right)-P\left(U_{\lambda_{n}}=x_{0}\right)\right|
\end{aligned}
$$




$$
+\left|P\left(U_{\lambda_{N}}=x_{0}\right)-P\left(U_{\lambda}=x_{0}\right)\right|
$$

Neammanee [4] showed that

$$
\begin{aligned}
\left|P\left(S_{n}=x_{0}\right)-P\left(U_{\lambda_{n}}=x_{0}\right)\right| & \leq \sup _{x \geq 1}\left|\Delta f_{x_{0}}(x)\right| \sum_{i=1}^{n} p_{i}^{2} \\
& \leq \min \left\{\frac{1-e^{-\lambda_{n}}}{\lambda_{n}}, \frac{1}{x_{0}}\right\} \sum_{i=1}^{n} p_{i}^{2}(\text { by }(2.4)) .
\end{aligned}
$$

Applying the proof detailed as in [1] and using (2.3), we have that

$$
\begin{aligned}
\left|P\left(U_{\lambda_{N}}=x_{0}\right)-P\left(U_{\lambda}=x_{0}\right)\right|= & \left|E\left\{\lambda f_{x_{0}}\left(U_{\lambda_{N}}+1\right)-U_{\lambda_{N}} f_{x_{0}}\left(U_{\lambda_{N}}\right)\right\}\right| \\
= & \mid E\left\{\lambda E\left\{\left[f_{x_{0}}\left(U_{\lambda_{N}}+1\right)\right] \mid \lambda_{N}\right\}\right. \\
& \left.-E\left\{\left[U_{\lambda_{N}} f_{x_{0}}\left(U_{\lambda_{N}}\right)\right] \mid \lambda_{N}\right\}\right\} \mid \\
= & \mid E\left\{\lambda E\left\{\left[f_{x_{0}}\left(U_{\lambda_{N}}+1\right)\right] \mid \lambda_{N}\right\}\right. \\
& \left.-\lambda_{N} E\left\{\left[f_{x_{0}}\left(U_{\lambda_{N}}+1\right)\right] \mid \lambda_{N}\right\}\right\} \mid \\
= & \left|E\left\{\left(\lambda-\lambda_{N}\right) E\left[f_{x_{0}}\left(U_{\lambda_{N}}+1\right) \mid \lambda_{N}\right]\right\}\right| \\
\leq & \sup _{x \geq 1}\left|f_{x_{0}}(x)\right| E\left|\lambda_{N}-\lambda\right| \\
\leq & \min \left\{\frac{1-e^{-\lambda}}{\lambda}, \frac{1}{x_{0}}\right\} E\left|\lambda_{N}-\lambda\right| .
\end{aligned}
$$

Taking the bounds in (3.3) and (3.4) to (3.2), hence (3.1) is obtained.

If $X_{i}$ 's are identically distributed, then the following corollary is an immediately consequence of the Theorem 3.1

Corollary 3.1. For $x_{0} \in \mathbb{N}$, if $p_{1}=p_{2}=\cdots=p$, then we have the following:

$$
\begin{aligned}
\left|P\left(S_{N}=x_{0}\right)-P\left(U_{\lambda}=x_{0}\right)\right| \leq & \min \left\{\frac{1-e^{-p E(N)}}{p E(N)}, \frac{1}{x_{0}}\right\} p E|N-E(N)| \\
& +\min \left\{E\left(1-e^{-p N}\right) p, \frac{E(N) p^{2}}{x_{0}}\right\} .
\end{aligned}
$$

Remark. Because

1. $\min \left\{\frac{1-e^{-\lambda}}{\lambda}, \frac{1}{x_{0}}\right\}<1$, 
2. $\min \left\{\frac{1-e^{-\lambda_{N}}}{\lambda_{N}}, \frac{1}{x_{0}}\right\} \leq \frac{1-e^{-\lambda_{N}}}{\lambda_{N}}$,

3. $\min \left\{\frac{1-e^{-\lambda}}{\lambda}, \frac{1}{x_{0}}\right\} E\left|\lambda_{N}-\lambda\right| \leq \frac{E\left|\lambda_{N}-\lambda\right|}{x_{0}}<\frac{2 \lambda}{x_{0}}$ and

4. $\min \left\{E\left(\frac{1-e^{-\lambda_{N}}}{\lambda_{N}} \sum_{i=1}^{N} p_{i}^{2}\right), \frac{1}{x_{0}} E\left(\sum_{i=1}^{N} p_{i}^{2}\right)\right\}<\frac{\lambda}{x_{0}}$.

Thus, the bounds in Theorem 3.1 is sharper than all bounds in (1.1)-(1.3).

\section{Conclusion}

In this study, a new bound for the point metric between the distribution of random sums of independent Bernoulli random variables and a Poisson distribution was obtained by using the Stein-Chen method. By comparing, this bound is sharper than those reported in [3]. Thus, the bound obtained in this study is more appropriate for measuring the accuracy of this pointwise approximation.

\section{References}

[1] A.D. Barbour, L. Holst, S. Janson, Poisson Approximation, Oxford Studies in Probability 2, Clarendon Press, Oxford, 1992.

[2] L.H.Y. Chen, Poisson approximation for dependent trials, Ann. Probab., 3 (1975), 534-545.

[3] S. Kongudomthrap, N. Chaidee, Bounds in Poisson approximation of random sums of Bernoulli random variables, Journal of Mathematics Research, 4 (2012), 29-35.

[4] K. Neammanee, Pointwise approximation of Poisson binomial by Poisson distribution. Stochastic Modelling and Applications, 6 (2003), 20-26.

[5] K. Teerapabolarn, A non-uniform bound in probability approximation via the Stein-Chen method, Stochastic Modelling and Applications, 9 (2006), $1-15$.

[6] K. Teerapabolarn, K. Neammanee, A non-uniform bound on Poisson approximation for dependent trials, Stochastic Modelling and Applications, $\mathbf{8}$ (2005), 17-31.

[7] R. Kun, K. Teerapabolarn, A pointwise Poisson approximation by $w$ functions, Applied Mathematical Sciences, 6 (2012), 5029-5037. 
[8] N. Yannaros, Poisson approximation for random sums of Bernoulli random variables, Statist. Probab. Lett., 11 (1991), 161-165. 